\title{
Color Image Compression Based on Directional All Phase Biorthogonal Transform
}

\author{
Chunxiao Zhang ${ }^{1}$, Chengyou Wang ${ }^{1 *}$ and Baochen Jiang ${ }^{1,2}$ \\ ${ }^{1}$ School of Mechanical, Electrical and Information Engineering, Shandong \\ University, Weihai 264209, China \\ ${ }^{2}$ School of Electronic Information Engineering, Tianjin University, Tianjin \\ 300072, China \\ sdwhzcx@126.com,wangchengyou@sdu.edu.cn, jbc@sdu.edu.cn
}

\begin{abstract}
There are images in which some directional edges other than the vertical or horizontal ones dominate. So the conventional discrete cosine transform (DCT) may not be the best choice for them. Directional DCT framework is able to provide a better coding performance for image blocks that contain directional edges. In addition, the all phase biorthogonal transform (APBT) algorithm can improve the performance of compression. In the light of directional DCT and APBT, directional APBT is proposed and applied to color image coding. Experimental results show that this framework can indeed improve the compression performance of color image remarkably.
\end{abstract}

Keywords: Color Image Compression; All Phase Biorthogonal Transform (APBT); Directional Discrete Cosine Transform (D-DCT); Directional APBT (D-APBT)

\section{Introduction}

Digital images and videos undoubtedly contribute a big portion to digital multimedia data that people are enjoying today. Nevertheless, in the raw format, each digital image or video data usually need a large volume of bits to represent, and therefore some sort of compression has to be done before it is transmitted or stored. Over the past three decades, many image and video coding methods have been developed for this compression task, such as predictive coding [1, 2], transform coding [3], vector quantization [4], and subband/wavelet coding [5, 6]. Among various coding techniques, the block-based transform approach has become particularly successful thanks to its simplicity, excellent energy compaction in the transform domain, super compromise between bit rate and quantization errors. Consequently, many international standards for image and video coding developed so far have adopted certain transform, including JPEG, H.261/H.263, MPEG1/2/4, and H.264. These coding standards almost cover all applications that we can enumerate from our daily life, such as digital cameras, video phones, internet media, digital TV broadcasting, and HDTV. With almost no exception, the transform chosen in these coding standards is the 2-D discrete cosine transform (DCT) [7, 8] that is applied on individual blocks of a square size $N \times N$. In practice, this conventional 2-D DCT is always implemented separately through two-point DCTs, along the vertical and horizontal directions. Although 2-D DCT has been so popular in image and video processing, it has some unpleasant defects. Once bit rate changes, more time is wasted in the complex multiplications for the complex quantization table. Additionally, the reconstructed image based on DCT has serious blocking artifacts at low bit rates. Considering these issues, Ref. [9] proposed the all phase digital filtering theory. The key idea of all phase digital filtering is to consider the all possible interception phase of data block in the process of the 
orthogonal transform. On this basis, the new concept of all phase Walsh biorthogonal transform (APWBT) was proposed in [10] and better coding performance was achieved when applied to grey image compression. Ref. [11] deduced the specific forms of all phase biorthogonal transform (APBT) matrices based on Walsh-Hadamard transform for subsequent application in still image compression. In APBT, uniform quantization value instead of complex quantization table is adopted, so the amount of computation decreases while changing bit rates. And the visual quality improves obviously at low bit rates for APBT algorithm.

In H.264 video standard, directional prediction is used. Considering this idea, Ref. [12] proposed a new block-based DCT framework-directional DCT (D-DCT) for image coding. In D-DCT, seven modes are proposed including the conventional DCT. Its basic idea is to choose the best transform-mode from all directional DCTs according to the direction of dominating edges within image. For the image in which vertical or horizontal directions are not dominating, D-DCT performs well. Experimental results show that rate distortion coding performance can be improved remarkably and certain coding gain is obtained.

In the light of directional DCT and APBT, directional APBT (D-APBT) is proposed in [13] and applied to grey image compression. Based on the previous work, we try to apply D-APBT to color image compression in this paper.

The remainder of this paper is organized as follows. In Section 2, we introduce the directional APBT algorithm. And the color image compression scheme is presented in Section 3. In Section 4, the experimental results and comparisons with D-DCT are given. Finally, conclusions are presented in Section 5.

\section{Directional APBT}

In H.264, there are in total eight directional prediction modes (the DC modeMode 2-is not counted) for blocks of size $4 \times 4$. Among these modes, one is the vertical prediction (Mode 0), one is the horizontal prediction (Mode 1), and the remaining are named as diagonal down-left (Mode 3), diagonal down-right (Mode 4), vertical-right (Mode 5), horizontal-down (Mode 6), vertical-left (Mode 7), and horizontal-up (Mode 8), respectively. This idea can be readily applied to any block size $N \times N$ to define the same eight directional modes. For instance, Figure 1 shows six directional modes (Modes 3 8) when $N=8$. It is easy to find that Mode 4 can be obtained by flipping Mode 3 either horizontally or vertically; Mode 6 can be obtained by transposing Mode 5, and Mode 7/8 can be obtained by flipping Mode $5 / 6$ either horizontally or vertically. To make our results general enough, we consider an arbitrary block size $N \times N$ and will first develop a truly directional APBT for the diagonal down-left mode, and then discuss the extension to other modes.

\subsection{Directional APBT for the Diagonal Down-Left Mode}

As shown in Figure 2, the first 1-D APBT will be performed along the diagonal down-left direction, i.e., for each diagonal line with $i+j=k, k=0,1, \cdots, 2 N-2$. There are in total $2 N-1$ diagonal down-left APBTs to be done, whose lengths are $\left[N_{k}\right]=[1,2, \cdots, N-1, N, N-1, \cdots, 2,1]$. After these APBTs, all the coefficients are expressed into a group of column vectors

$$
\boldsymbol{Y}_{k}=\left[Y_{0, k}, Y_{1, k}, \cdots, Y_{N_{k}-1, k}\right]^{\mathrm{T}}, k=0,1, \cdots, 2 N-2
$$

Notice that each column of $\boldsymbol{Y}_{k}$ has a different length $N_{k}$, with the DC component placed at top, followed by the first AC component and so on; see Figure 3 for $N=8$. 


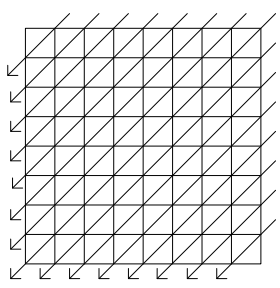

(a)

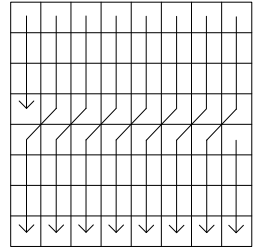

(d)

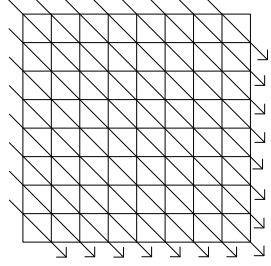

(b)

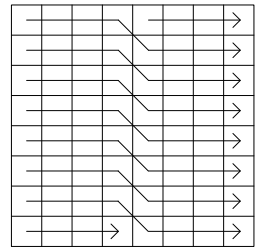

(e)

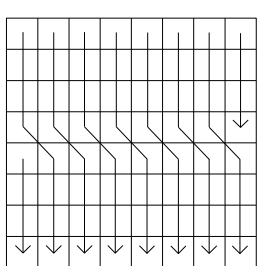

(c)

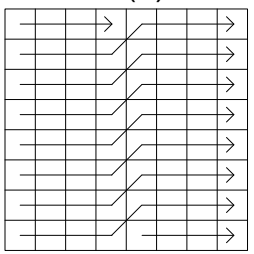

(f)

Figure 1. Six Directional Modes Defined in a Similar Way as was Used in Directional DCT for the Block Size 8x8: (a) Mode 3, (b) Mode 4, (c) Mode 5, (d) Mode 6, (e) Mode 7, (f) Mode 8

Next, the second 1-D APBT is applied to each row that can be expressed as $\left[Y_{u, v}\right]_{v=0: 2 N-2-2 u}$ for $u=0,1, \cdots, N-1$. The coefficients after the second APBT are pushed horizontally to the left and denoted as $\left[\hat{Y}_{u, v}\right]_{v=u: 2 N-2-u}$ for $u=0,1, \cdots, N-1$. The right sub-image of Figure 3 shows a modified zig-zag scanning that will be used to convert the 2-D coefficient block into a 1-D sequence so as to facilitate the variable length coding based on run length.

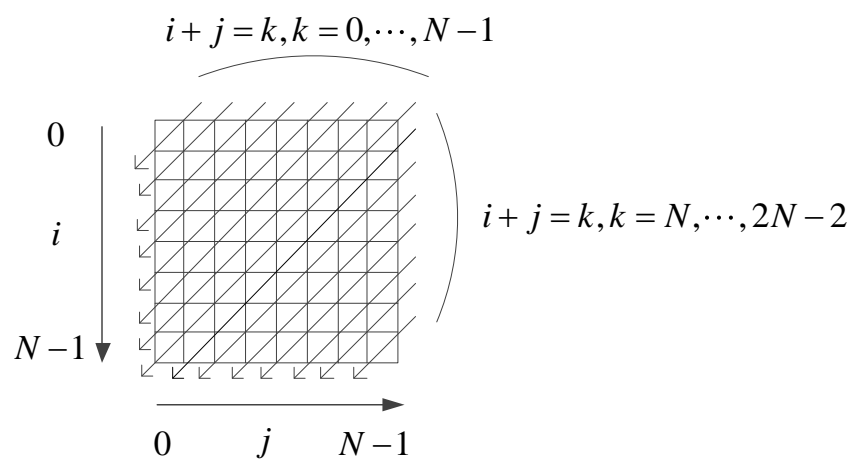

Figure 2. $N \times N$ Image Block in which the First 1-D APBT will be Performed along the Diagonal Down-left Direction

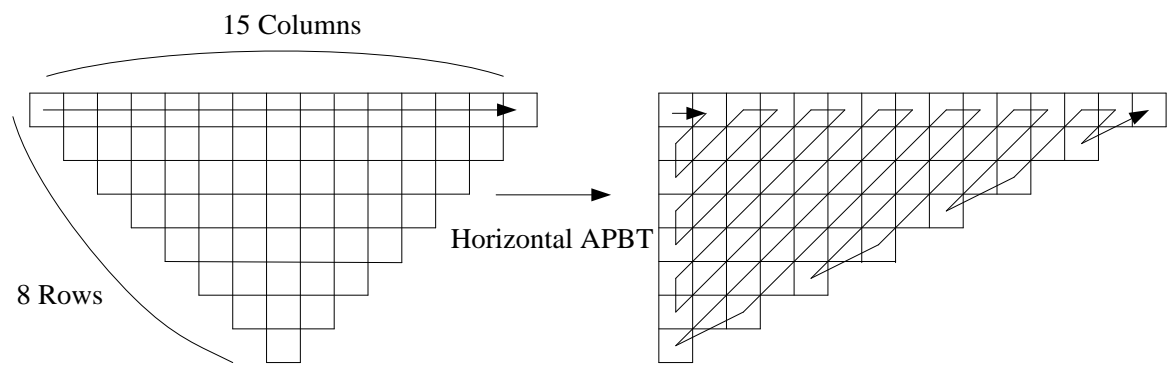

Figure 3. Example of $N=8$ : Arrangement of Coefficients after the First APBT (left) and Arrangement of Coefficients After the Second APBT as Well as the Modified Zig-zag Scanning (right) 


\subsection{Extension to Other Directional Modes}

Extension to other directional modes is straightforward. For instance, for the diagonal down-right mode (Mode 4), we can simply flip it, and then the flipped image block will fall into the case as discussed above. For Modes 6 8, we can simply flip or transpose the image block first and then the manipulated block will fall into the case of Mode 5. Furthermore, Modes 0 and 1 are the conventional APBT and thus produce exactly the same result, we need to consider seven modes only.

\section{Color Image Compression Scheme}

The standard of JPEG can compress the source color image data (luminance component and chrominance components) from the different color spaces like RGB, $\mathrm{YCbCr}$, etc. Wherein, RGB model almost includes all of the colors perceived by the human eyes. However, this model is not suitable for graphical analysis, since the R, $\mathrm{G}$ and $\mathrm{B}$ components are highly relevant. When the brightness is changed, the three components will be amended accordingly [14]. Therefore, most of the color image compression schemes firstly transform the original image data from highly correlated RGB color space into a decorrelated color space like YUV, YCbCr, etc., [15]. Then the redundancies between the color components are reduced, and we can code the decorrelated color components by JPEG image compression schemes.

Figure 4 shows the procedure of compression for color image in this paper. Firstly, we transform the original data from RGB color space into $\mathrm{YCbCr}$ color space through Eq. (2), separating the luminance component and chrominance components. Then the separated component is processed by JPEG standard.

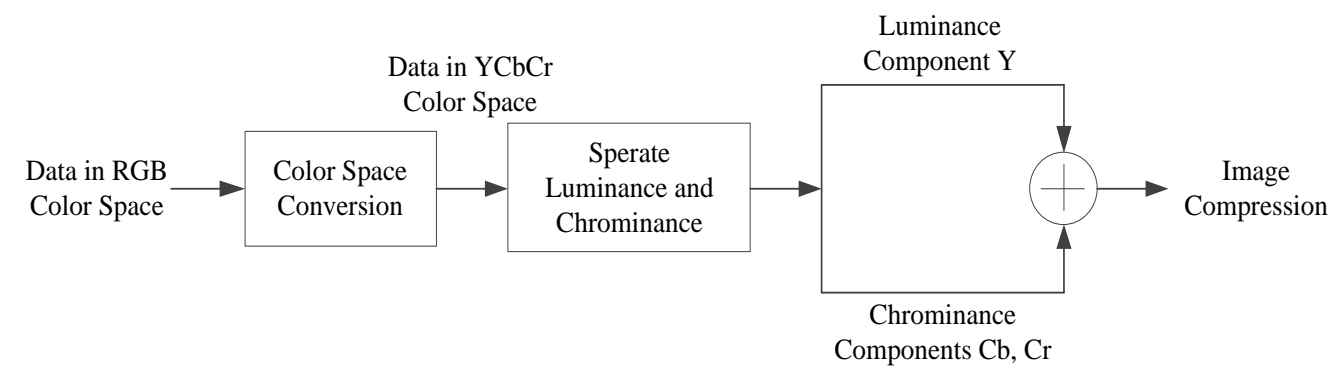

Figure 4. Separation of Luminance and Chrominance

$$
\left\{\begin{array}{l}
\mathrm{Y}=0.2990 \mathrm{R}+0.5870 \mathrm{G}+0.1140 \mathrm{~B} \\
\mathrm{Cb}=-0.1687 \mathrm{R}-0.3313 \mathrm{G}+0.5 \mathrm{~B}+128 \\
\mathrm{Cr}=0.5000 \mathrm{R}-0.4187 \mathrm{G}-0.0813 \mathrm{~B}+128
\end{array}\right.
$$

Both the luminance and chrominance components could be processed by the procedure presented in Figure 5. We'll take the luminance as an example to illustrate the scheme in the following part. When luminance data are got, choose the best mode from seven modes according to the criterion of minimum mean-square error (MSE). In the sending part, when calculating the MSE, both of D-APBT and quantization are needed, so they are combined to one step in Figure 5. Additionally, uniform value is adopted in the procedure of quantization. After the luminance block is transformed and quantized in the best mode, the remaining steps are same with DCT-JPEG: zig-zag scanning and entropy encoding. Then the compressed data are transmitted through the channel. In the receiving part, all the procedures are completely opposite to the sending part. Through the inverse color-space-conversion, the reconstructed color image is got. 


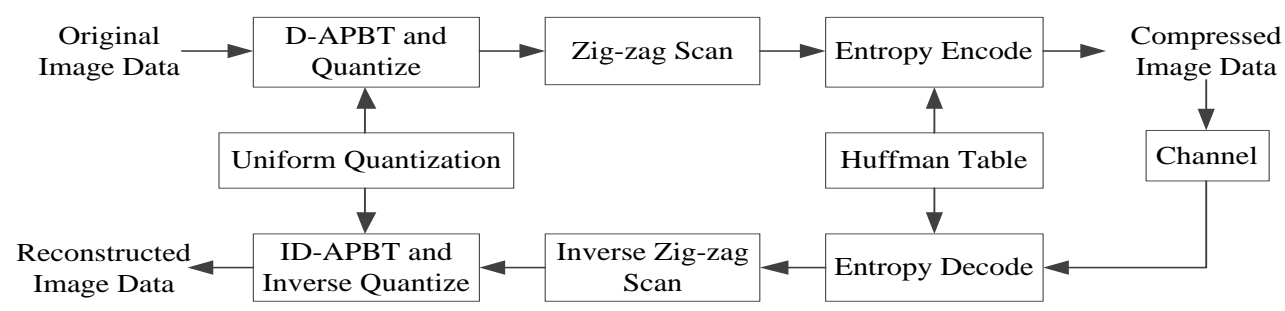

Figure 5. Directional APBT-JPEG Image Compression Procedure

\section{Experimental Results}

To measure the performance of algorithm proposed in this paper, we choose the Composite Peak Signal to Noise Ratio (CPSNR) defined as

$$
\text { CPS N R }=10 \log _{10}\left|\frac{255^{2}}{\frac{1}{3 M N} \sum_{k=1}^{3} \sum_{i=1}^{M} \sum_{j=1}^{N}\left[I_{\text {in }}(i, j, k)-I_{\text {out }}(i, j, k)\right]^{2}}\right|(\mathrm{d} \mathrm{B})
$$

where $I_{\text {in }}$ and $I_{\text {оu }}$ are the original and reconstructed images respectively, $M$ and $N$ are the dimensions of each color component array, $i$ and $j$ are the locations of pixels in the color plane, and $k$ represents the color plane.

In this part, the seven directional modes referred to above are considered. What we need to do is to code each image block with the most appropriate directional mode. The simulation adopts the exhaustive traversal method, that is, seven modes are used to code each block separately, then chooses the best direction mode according to the rate distortion. Firstly, one bit is used to distinguish the conventional mode and directional mode, and then the extra three bits are used to distinguish Modes 3 8.

The experimental results are given as follows. The simulation environment is MATLAB 7.0. The color images of Peppers and Lena with size of $512 \times 512$ are chosen as the test images. When RGB components are transformed into $\mathrm{YCbCr}$ space, the signal-mode of 4:4:4 is adopted. Therefore, sampling and interpolation are not needed. For luminance and chrominance blocks, the same quantization value is adopted. Table 1 and Table 2 show the experimental results of directional DCT, directional APDCBT and directional APIDCBT applied to image Peppers and Lena respectively. To be more clear and intuitive, we draw the ratio distortion curves. As shown in Figure 6, at low bit rates, D-APBT algorithm performs better than D-DCT and at high bit rates, D-APBT algorithm performs close to D-DCT.

Table 1. CPSNR Comparison of D-DCT and D-APBT Applied to Image Peppers

\begin{tabular}{|c|c|c|c|}
\hline \multirow{2}{*}{ Bit rate/bpp } & \multicolumn{3}{|c|}{ CPSNR/dB } \\
\cline { 2 - 4 } & D-DCT & D-APDCBT & D-APIDCBT \\
\hline 0.26 & 17.90 & 20.84 & 20.90 \\
\hline 0.30 & 22.12 & 23.65 & 23.93 \\
\hline 0.35 & 24.27 & 25.33 & 25.77 \\
\hline 0.40 & 25.73 & 26.41 & 26.91 \\
\hline 0.50 & 27.82 & 27.92 & 28.38 \\
\hline 0.60 & 29.02 & 28.83 & 29.32 \\
\hline 0.70 & 29.82 & 29.65 & 30.09 \\
\hline 0.80 & 30.37 & 30.25 & 30.63 \\
\hline
\end{tabular}


Table 2. CPSNR Comparison of D-DCT and D-APBT Applied to Image Lena

\begin{tabular}{|c|c|c|c|}
\hline \multirow{2}{*}{ Bit rate/bpp } & \multicolumn{3}{|c|}{ CPSNR/dB } \\
\cline { 2 - 4 } & D-DCT & D-APDCBT & D-APIDCBT \\
\hline 0.30 & 22.48 & 24.79 & 24.87 \\
\hline 0.35 & 26.36 & 28.24 & 28.27 \\
\hline 0.40 & 28.69 & 29.39 & 29.55 \\
\hline 0.50 & 30.59 & 30.89 & 31.09 \\
\hline 0.60 & 31.70 & 31.74 & 32.03 \\
\hline 0.70 & 32.59 & 32.67 & 32.98 \\
\hline 0.80 & 33.31 & 33.29 & 33.61 \\
\hline 0.90 & 33.85 & 33.74 & 34.13 \\
\hline
\end{tabular}

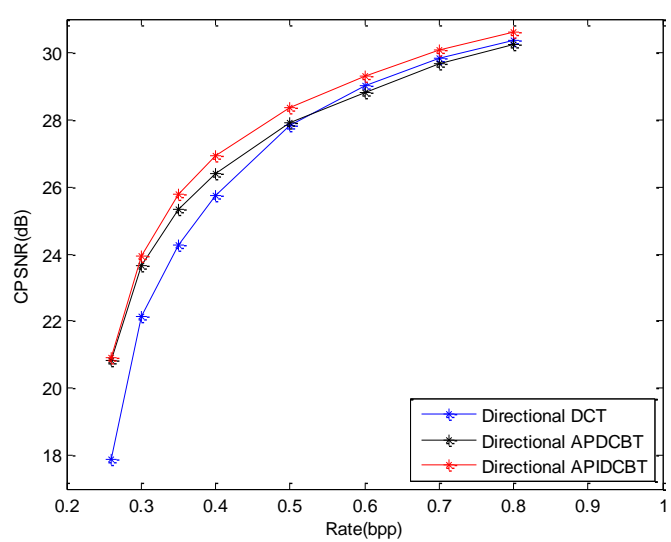

(a)

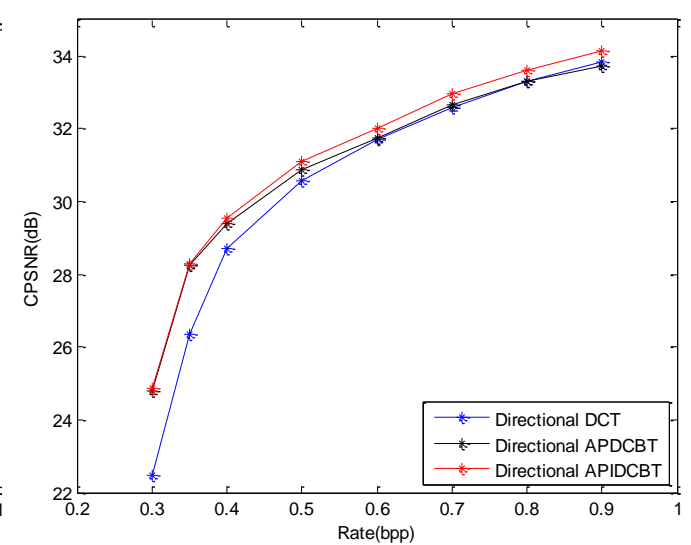

(b)

Figure 6. Rate Distortion Curves: (a) Peppers, (b) Lena

To evaluate the subjective effect, the reconstructed images of Peppers at $0.40 \mathrm{bpp}$ obtained by using D-DCT and D-APBT algorithms are presented in Figure 7. Compared with Figure 7(b) with D-DCT, the blocking artifacts of Figure 7(c) with D-APDCBT and Figure 7(d) with D-APIDCBT reduce obviously.

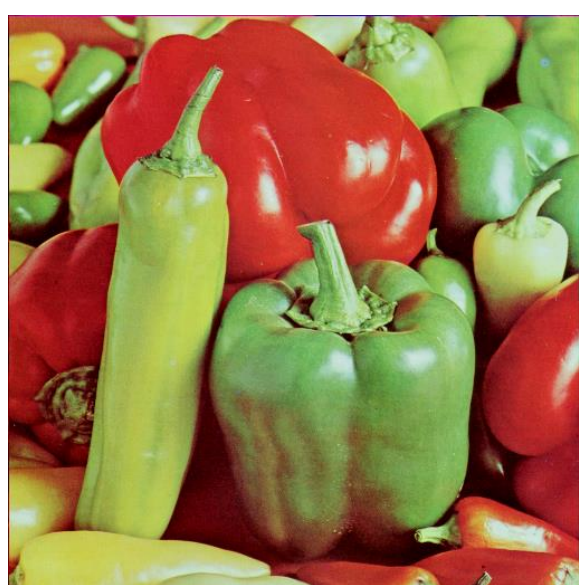

(a)

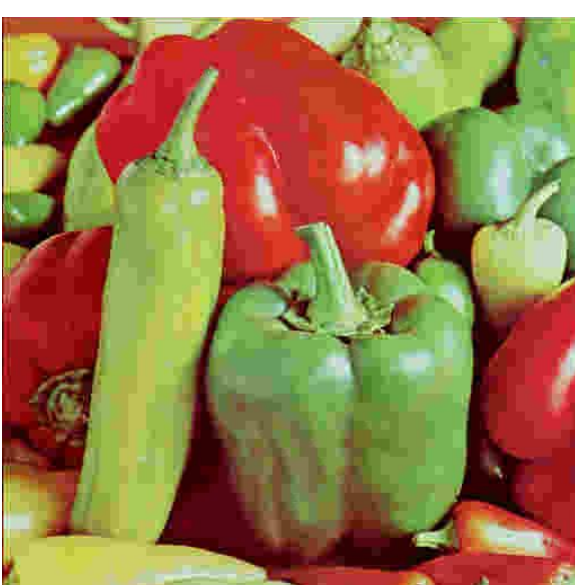

(b) 


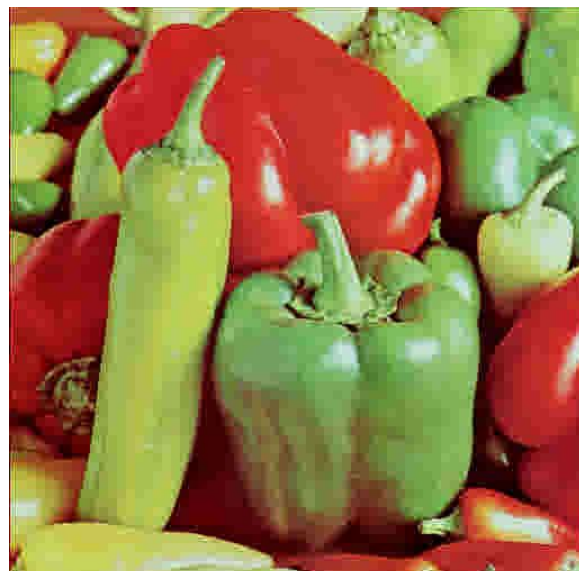

(c)

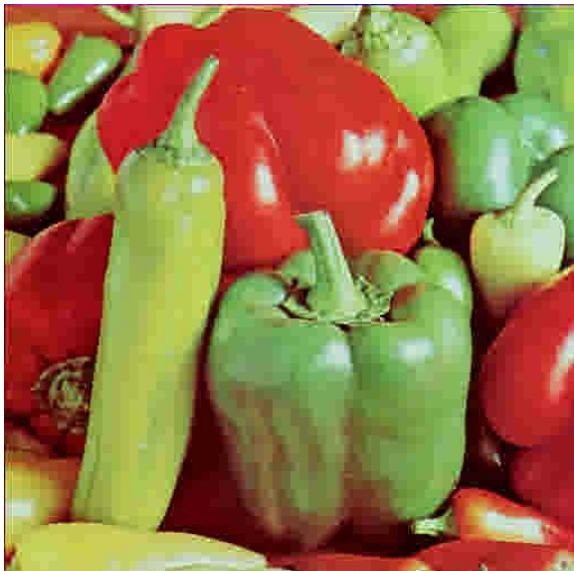

(d)

Figure 7. Original and Reconstructed Images Peppers (0.40bpp): (a) Original Image, (b) D-DCT (25.73dB), (c) D-APDCBT (26.41dB), (d) DAPIDCBT (26.91dB)

\section{Conclusion}

In this paper, the D-APBT algorithm is applied to color image coding. Compared with the D-DCT, the D-APBT algorithm performs better at low bit rates. And even at high bit rates, it performs close to D-DCT. Since uniform value is used in the step of quantization, the computational complexity decreases obviously. So the D-APBT algorithm could be successfully applied in color image compression. It not only extends the application range of the APBT theory, but also proves the effectiveness of the directional transform theory.

Although better performance has been achieved in APBT algorithm, the procedure of choosing the best mode still needs an amount of calculation. Hence, the further work can be focused on exploring a better method to choose the appropriate mode.

\section{Acknowledgements}

This work was supported by the Graduate Innovation Foundation of Shandong University at WeiHai (GIFSDUWH) (Grant No. yjs12028), the promotive research fund for excellent young and middle-aged scientists of Shandong Province, China (Grant No. BS2013DX022) and the National Natural Science Foundation of China (Grant No. 61201371). The authors would like to thank Xiaoyan Wang, Fanfan Yang and Qiming Fu for their kind help and valuable suggestions. The authors also thank the anonymous reviewers and the editors for their valuable comments to improve the presentation of the paper.

\section{References}

[1] L. R. Rabiner and R. W. Schafer, "Digital Processing of Speech Signals", Englewood Cliffs, NJ: Prentice-Hall, (1978).

[2] N. S. Jayant and P. Noll, "Digital Coding of Waveforms", Englewood Cliffs, NJ: Prentice-Hall, (1984).

[3] R. J. Clark, "Transform Coding of Images", London, U. K.: Academic, (1985).

[4] A. Gersho and R. M. Gray, "Vector Quantization and Signal Compression", Boston, MA: Kluwer, (1991).

[5] P. P. Vaidyanathan, "Multirate Systems and Filter Banks", Englewood Cliffs, NJ: Prentice-Hall, (1993).

[6] M. Vetterli and J. Kovacevic, "Wavelets and Subband Coding", Englewood Cliffs, NJ: Prentice-Hall, (1995).

[7] N. Ahmed, T. Natarajan and K. R. Rao, "Discrete cosine transform", IEEE Transactions on Computer, vol. 23, no. 1, (1974), pp. 90-93. 
[8] K. R. Rao and P. Yip, "Discrete Cosine Transform-Algorithms, Advantages, Applications", London, U.K.: Academic, (1990).

[9] Z. X. Hou and X. Yang, "The all phase DFT filter", Proceedings of the 10th IEEE Digital Signal Processing Workshop and the 2nd IEEE Signal Processing Education Workshop, Pine Mountain, (2002); Georgia, USA.

[10] C. Y. Wang, Z. X. Hou and A. P. Yang, "Image coding algorithm based on all phase Walsh biorthogonal transform", Proceedings of the IEEE International Symposium on Microwave, Antenna, Propagation and EMC Technologies for Wireless Communications, (2007); Hangzhou, China.

[11] Z. X. Hou, C. Y. Wang and A. P. Yang, "All phase biorthogonal transform and its application in JPEGlike image compression”, Signal Processing: Image Communication, vol. 24, no. 10, (2009), pp. 791802.

[12] B. Zeng and J. J. Fu, "Directional discrete cosine transforms-A new framework for image coding", IEEE Transactions on Circuits and Systems for Video Technology, vol. 18, no. 3, (2008), pp. 305-313.

[13] C. Y. Wang, "Directional APBT and its application in image coding", Proceedings of the IEEE 11th International Conference on Signal Processing, vol. 1, (2012), pp. 728-731.

[14] G. Chen, "Application of processing techniques from color image to grey image", Proceedings of the 2nd International Conference on Software Technology and Engineer, (2010); San Juan, PR, USA.

[15] V. K. Nath, D. Hazarika and A. Mahanta, "A novel approach to color image compression using 3-D discrete cosine transform", Proceedings of the IEEE International Workshop on Machine Learning for Signal Processing, (2008); Cancn, Mexico.

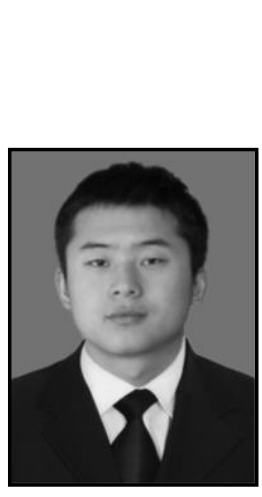

\section{Authors}

Chunxiao Zhang was born in Shandong province, China in 1990. He received his B.S. degree in electronic information science and technology from Shandong University, Weihai, China, in 2012. Now he is pursuing his M.E. degree in circuits and systems in Shandong University, Weihai, China. His research interests concentrate on image and video processing.

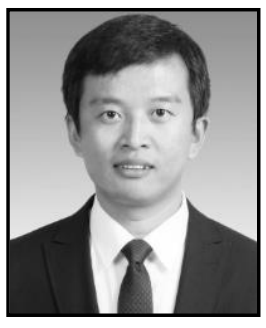

Chengyou Wang was born in Shandong province, China in 1979. $\mathrm{He}$ received his B.E. degree in electronic information science and technology from Yantai University, China, in 2004 and his M.E. and $\mathrm{Ph} . \mathrm{D}$. degree in signal and information processing from Tianjin University, China, in 2007 and 2010 respectively. Now he is an associate professor in the School of Mechanical, Electrical and Information Engineering, Shandong University, Weihai, China. His current research interests include image processing and transmission techniques, multidimensional signal and information processing, and smart grid technology.

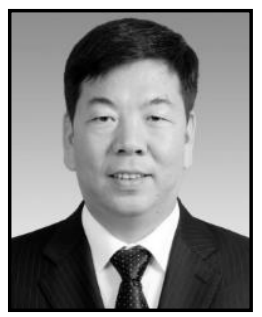

Baochen Jiang was born in Shandong province, China in 1962. $\mathrm{He}$ received his B.S. degree in radio electronics from Shandong University, China, in 1983 and his M.E. degree in communication and electronic systems from Tsinghua University, China, in 1990. Now he is a professor in the School of Mechanical, Electrical and Information Engineering, Shandong University, Weihai, China. His current research interests include signal and information processing, image and video processing, and smart grid technology. 